\title{
Análisis del proceso de enseñanza y de aprendizaje de los contenidos de la asignatura de Geografía en nivel primaria en México
}

\author{
Analysis of the teaching and learning process \\ of the subject contents of Geography in Mexico primary school \\ Marco Antonio Bernal Díaz \\ Diana Carolina Fonseca Polanco \\ Aníbal Zaldívar Colado
}

\begin{abstract}
RESUMEN
Esta investigación tiene como objetivo analizar el estudio de la asignatura de Geografía en nivel primaria, donde el niño inicia la comprensión de la Tierra, su superficie, los paisajes, así como lugares y regiones por medio de exploración documental. Para esta pesquisa se emplearon técnicas de investigación necesarias para la recolección de datos como el análisis documental, observación dentro del contexto del aula, encuesta y entrevista. Los datos fueron analizados cualitativa y cuantitativamente, para conocer características, estrategias y los recursos utilizados por los alumnos en el aula, así como la percepción docente en su quehacer como agente del conocimiento. Entre los resultados más relevantes se encontró que los docentes utilizan principalmente estrategias tradicionales de enseñanza, como el libro de texto y el cuaderno, y los estudiantes no se sienten motivados con estos medios, además de preferir aprender con el uso de nuevas tecnologías. La información obtenida de esta investigación servirá en un futuro como base para la etapa de análisis del desarrollo de un software que sirva como estrategia tecnológica en la asignatura de Geografía para fortalecer las competencias de los discentes y docentes.
\end{abstract}

Palabras clave: aprendizaje, enseñanza, geografía, nivel básico.

\begin{abstract}
This research aims to analyze the study of the subject of Geography in an elementary school setting, where the child begins to understand the Earth, its surface, landscapes, as well as places and regions through documentary exploration. For this research, techniques necessary for data collection were used, such as documentary analysis, observation within the classroom context, survey, and interview. The data were analyzed qualitatively and quantitatively, to know the characteristics, strategies, and resources used by the students in the classroom, as well as the teachers' perception in their work as an agent of knowledge. Among the most relevant results, it was found that teachers mainly use traditional teaching strategies, such as the textbook and notebook, and the students do not feel motivated by these means, besides preferring to learn with the use of new technologies. The information obtained from this research will serve in the future as a basis for the analysis stage of the development of software that serves as a technological strategy in the subject of Geography to strengthen the competencies of students and teachers.
\end{abstract}

Keywords: learning, teaching, geography, elementary school. 


\section{INTRODUCCIÓN}

Actualmente el docente de nivel básico debe ver su rol de instructor como alguien que no únicamente desarrolla el conocimiento plasmado en los planes y programas de estudio, sino que también despierta un verdadero interés y entusiasmo por esos conocimientos. El profesor de Geografía de nivel primaria tiene que ser consciente de la importancia de esta dentro del currículo y de su propio papel como experto capacitador y motivador para los alumnos. Por tal motivo, debe concientizar a los niños que están en condiciones de hacer Geografía, porque viven en el mundo; negocian e interactúan con una variedad de paisajes (humanos y naturales) a diario. Por ejemplo, tienen todas las rutas y vacaciones planificadas; todos se han detenido a admirar una vista, y han tomado decisiones sobre dónde vivir, trabajar y jugar (Martin, 2006). A través de estas interacciones y decisiones diarias, habrán acumulado una amplia base de conocimiento sobre el mundo, ya sea de experiencias de primera o segunda mano, reconociéndolo como útil, lo que contrasta con la afirmación de Blankman, Schoonenboom, Van der Schee, Boogaard y Volman (2016), quienes aseguran que los estudiantes que se preparan para convertirse en maestros de escuela primaria parecen tener poca conciencia de los conceptos básicos de la geografía (enseñanza).

No puede dejarse de lado que los niños y jóvenes del siglo XXI viven en una era digital donde utilizan las tecnologías de información y comunicación (TIC) desde temprana edad con distintos fines, ellos son conocidos como nativos digitales, debido a que piensan y procesan la información de una manera diferente y su destreza en el manejo de las tecnologías es superior a la de sus profesores denominados inmigrantes digitales. Los inmigrantes digitales se comunican de modo diferente con sus propios hijos, ya que se ven en la obligación de "aprender de una nueva lengua" que sus vás-

Marco Antonio Bernal Díaz. Investigador independiente, Sinaloa, México. Es maestro en Ciencias en Sistemas de Información Administrativa por la Universidad de Occidente y doctor en Educación por parte de la Universidad del Pacífico Norte. Cultiva la línea de investigación "Software educativo y tecnología educativa". Docente de la licenciatura en Sistemas Computacionales en la Universidad de Occidente Unidad Regional Mazatlán en los periodos 2012 y 2015. Correo electrónico: markobernal@, hotmail.com. ID: https://orcid.org/0000-0003-3926-6837.

Diana Carolina Fonseca Polanco. Profesora de asignatura B en la Universidad Autónoma de Occidente, Unidad Regional Mazatlán, Sinaloa, México. Es miembro investigador del Verano de Investigación Científica y Tecnológica del Pacífico. Es maestra en Ciencias en Sistemas de Información Administrativa por la Universidad de Occidente y doctora por parte de la Universidad de Durango. Cultiva la línea de investigación "Tecnología educativa”. Docente de la Ingeniería en Software. Correo electrónico: dianafonsecapolanco@yahoo.com.mx. ID: https://orcid.org/0000-0003-3232-8580.

Aníbal Zaldívar Colado. Profesor investigador de tiempo completo titular C de la Universidad Autónoma de Sinaloa, México. Entre sus publicaciones recientes se encuentran el libro Análisis de redes sociales, estrategias de empleo y de desarrollo local (2020) y el artículo "Análisis crítico de ambientes virtuales de aprendizaje" en la revista Utopía y Praxis Latinoamericana. Cultiva la línea de investigación “Tecnología educativa”. Análisis de datos en investigación educativa. Correo electrónico: azaldivar@uas.edu.mx. ID: https:// orcid.org/0000-0002-6622-6630. 
tagos conocen y dominan como nativos; lengua que, además, ha pasado a instalarse en su cerebro (Prensky, 2010).

En la actualidad el uso de las TIC se encuentra inmerso en todas nuestras actividades de la vida cotidiana (personales, laborales, profesionales, etc.) y está teniendo un impacto significativo en la vida social, económica y cultural de la sociedad contemporánea (Santillán, 2006).

Esto indica que las necesidades requeridas en el mundo actual son de individuos capaces de adaptarse rápidamente al mundo electrónico, donde los avances científicos van a un ritmo muy acelerado y se encuentran inmersos en las actividades que se realizan hoy en día.

Ante esto, el gobierno de México ha realizado diversos cambios en el sistema escolar para contar con un modelo educativo que se adapte a las nuevas necesidades, en él se menciona que el elemento indispensable para el desarrollo de las competencias del siglo XXI es la incorporación de las TIC al aprendizaje, debido a que son consideradas como una herramienta clave para participar en las dinámicas de las sociedades contemporáneas, e investigar, resolver problemas, producir contenidos didácticos, expresar ideas e innovar (SEP, 2017). Es común encontrar en las escuelas clases monótonas, tal y como se realizaban en el pasado, en las que se puede observar que los docentes siguen utilizando las mismas prácticas y métodos tradicionales en la mayoría de las asignaturas. Por ello, es preocupante que los estudiantes consideren una materia tan relevante e importante como Geografía, aburrida, tediosa y cansada al utilizar los mismos recursos tradicionales, al ser la Geografía una asignatura importante, en la cual los estudiantes van conociendo paulatinamente el paisaje local, su historia, cultura, belleza natural y atractivos, para adquirir un sentido de orgullo y pertenencia a su tierra natal (Tomcikova y Rakytova, 2018).

Si bien los recursos didácticos habituales empleados en la mayoría de las asignaturas han dado resultado, sigue siendo común encontrar desinformación y nociones equivocadas sobre la Geografía, porque las prácticas de enseñanza y de aprendizaje en la educación primaria basan su proceso en la memorización. Este método ha sido criticado por algunos educadores, si bien no se oponen a esta forma de enseñar, pero sí a su abuso como fórmula de enseñanza. De la misma manera se resistían al uso exclusivo del texto, pues lo consideraban necesario, pero únicamente como auxiliar de maestros y alumnos (Lima, Bonilla y Arista, 2010). Por tal motivo, los docentes deben emplear estrategias para crear nuevos ambientes de aprendizaje que involucren las TIC como apoyo en el desarrollo de conocimientos, habilidades, actitudes y valores; procurando el uso correcto de estas herramientas, debido a que son utilizadas por los alumnos para juegos, redes sociales, etc.

Considerando lo anterior, surge la motivación de analizar el estudio de la asignatura de Geografía en nivel primaria, tanto desde la perspectiva docente como del discente, que describa la enseñanza y el aprendizaje de esta materia. Esta investiga- 
ción es una primera etapa que será la base para la segunda, los resultados obtenidos proporcionarán los datos necesarios para la fase de análisis del desarrollo de un futuro diseño y creación de un software que cuente con las funciones y características requeridas por alumnos y maestros.

\section{El sistema educativo en México}

El sistema educativo en México ha tenido varias transformaciones a lo largo del tiempo, entre ellas la Reforma Integral de Educación Básica (RIEB), para la cual se desarrolló una política pública orientada a elevar la calidad educativa, que favorece la articulación en el diseño y desarrollo del currículo para la formación de los estudiantes de preescolar, primaria y secundaria, colocando en el centro del acto educativo al discente, el logro de los aprendizajes, los estándares curriculares y el desarrollo de las competencias que le permitan al alumno alcanzar el perfil de egreso de la educación básica (SEP, 2011a).

La RIEB consistió en reformas curriculares en los siguientes tres niveles (SEP, 2017):

1. En el 2004 la reforma de Educación Preescolar.

2. En el 2006 la reforma de la Educación Secundaria.

3. En el 2009 la reforma de Educación Primaria.

Esta serie de reformas llevó a la creación del Nuevo Modelo Educativo para la educación obligatoria con la finalidad de mejorar la calidad y la equidad académica para formar integralmente y lograr los aprendizajes que se necesitan para desarrollar con éxito sus proyectos de vida en un mundo globalizado, como lo exige la sociedad del siglo XXI (SEP, 2017). La finalidad de este cambio de paradigma pedagógico es desarrollar individuos capaces de adaptarse a entornos cambiantes y diversos; de pensamiento complejo, críticos, creativos, reflexivos y flexibles, y que resuelvan problemas de manera innovadora. Este modelo educativo resalta en uno de sus ámbitos las habilidades digitales que debe tener cada uno de los estudiantes al culminar determinado nivel educativo, cumpliendo así con el perfil de egreso, en el cual se define la progresión mínima común de lo aprendido por los discentes (SEP, 2017), el centro y referente fundamental del aprendizaje es el estudiante, porque desde etapas tempranas se requiere generar su disposición y capacidad de continuar aprendiendo a lo largo de su vida, desarrollar habilidades superiores del pensamiento para solucionar problemas, pensar críticamente, comprender y explicar situaciones desde diversas áreas del saber, manejar información, innovar y crear en distintos órdenes de la vida (SEP, 2011b).

En este sentido, es necesario reconocer que existe una diversidad social, cultural, lingüística, de capacidades y estilos de aprendizaje de cada discente, lo cual lleva al docente a comprender cómo aprende y generar un ambiente propicio para la generación de conocimiento significativo de interés. 


\section{La geografía en educación básica}

Actualmente la asignatura Geografía en educación primaria da continuidad a los aprendizajes de los alumnos en relación con el entorno donde viven para que reconozcan la distribución y las relaciones de los componentes naturales, culturales, económicos y políticos de la geografía desde lo local hasta lo global, mediante el desarrollo integrado de conceptos, habilidades y actitudes; valorar la diversidad natural, social, lingüística y económica, y participar en situaciones de la vida cotidiana para el cuidado del ambiente y la prevención de desastres (SEP, 2011c). Esto no ha cambiado mucho, desde el siglo XIX ha dado a conocer las peculiaridades del espacio geográfico a escala local, estatal, nacional y mundial; participa del estudio de las ciencias naturales como de las sociales y pone gran énfasis en las relaciones espaciales de las diferentes expresiones de la naturaleza y la sociedad. De ahí su relevancia como ciencia y como asignatura escolar en todos los niveles educativos del país (Castañeda, 2005).

La geografía, como disciplina científica, tiene un pasado muy lejano: en la antigua Grecia ya se conocía y estudiaba como tal, precisamente ahí tuvo su origen y se desarrolló como ciencia. Con el paso del tiempo, y en correspondencia con los paradigmas dominantes de los diferentes periodos históricos (Edad Antigua, Edad Media, Renacimiento, Edad Moderna y Edad Contemporánea), ha justificado su incorporación y estudio en el currículo escolar (SEP, 2011d, pp. 1).

Cada una de las asignaturas contempladas en el mapa curricular cumple con un objetivo, tal y como lo marca el Plan de Estudios 2011. Los propósitos del estudio de la Geografía para la educación básica son los siguientes (SEP, 2011b):

a) Explicar relaciones entre componentes naturales, sociales, culturales, económicos y políticos del espacio geográfico para construir una visión integral del espacio en las escalas local, estatal, nacional, continental y mundial.

b) Movilizar conceptos, habilidades y actitudes en situaciones de la vida cotidiana para asumirse como parte del espacio geográfico, valorar la diversidad natural, social, cultural y económica, y fortalecer la identidad nacional.

c) Participar de manera informada, reflexiva y crítica en el espacio donde se habita para el cuidado y la conservación del ambiente, así como para contribuir a la prevención de desastres.

Los propósitos de estudio de la Geografía para la educación son (SEP, 2011b):

a) Reconocer la distribución y las relaciones de los componentes naturales, sociales, culturales, económicos y políticos del espacio geográfico para caracterizar sus diferencias en las escalas local, estatal, nacional, continental y mundial.

b) Adquirir conceptos, habilidades y actitudes para construir la identidad nacional mediante el reconocimiento de la diversidad natural, social, cultural y económica del espacio geográfico. 
c) Participar de manera informada en el lugar donde se vive para el cuidado del ambiente y la prevención de desastres.

El mapa curricular del sistema educativo se organiza a partir de aprendizajes claves, entendidos como un conjunto de contenidos, actitudes, habilidades y valores fundamentales que contribuyen sustancialmente al crecimiento de la dimensión intelectual, personal y social del estudiante y que se desarrollan de manera significativa en la escuela (SEP, 2017). Los aprendizajes clave se dividen en tres componentes que están contemplados en todos los niveles educativos (preescolar, primaria y secundaria), cada uno de ellos integrado por asignaturas, áreas o ámbitos.

El primer componente curricular, denominado Campos de formación académica, se encuentra divido en tres campos:

1. Lenguaje y comunicación.

\subsection{Preescolar}

1.1.1. Lenguaje y comunicación (1, 2 y 3)

1.1.2. Inglés (preescolar 3)

1.2. Primaria

1.2.1. Lengua materna $(1,2,3,4,5$ y 6$)$

1.2.2. Español. Segunda lengua (1, 2, 3, 4, 5 y 6$)$

1.2.3. Lengua extranjera. Inglés $(1,2,3,4,5$ y 6$)$

1.3. Secundaria

1.3.1. Español $(1,2$ y 3$)$

1.3.2. Inglés $(1,2$ y 3$)$

2. Pensamiento matemático

2.1. Matemáticas (preescolar, primaria y secundaria)

3. Exploración y comprensión del mundo natural y social

3.1. Preescolar

3.1.1. Exploración del mundo natural y social (1, 2 y 3$)$

\subsection{Primaria}

3.2.1. Conocimiento del medio (1 y 2)

3.2.2. Ciencias Naturales y Tecnología (3, 4 ,5 y 6 )

3.2.3. Historias, paisajes y convivencia (3)

3.2.4. Geografía (4, 5 y 6$)$

3.2.5. Formación Cívica y Ética $(4,5$ y 6$)$

3.2.6. Historia $(4,5$ y 6$)$

3.3. Secundaria

3.3.1. Biología (1)

3.3.2. Geografía (1)

3.3.3. Física (2)

3.3.4. Química (3) 


\subsubsection{Formación Cívica y Ética $(1,2$ y 3)}

3.3.6. Historia $(1,2$ y 3$)$

El segundo componente, llamado Área de desarrollo personal y social, está divido en tres áreas:

1. Artes (preescolar, primaria y secundaria).

2. Educación socioemocional (preescolar, primaria y secundaria, en este último nivel educativo cambia a Tutoría y educación emocional).

3. Educación física (preescolar, primaria y secundaria).

Por último, el tercer componente, denominado Ámbito de autonomía curricular, se divide en cinco ámbitos en los tres niveles educativos (preescolar, primaria y secundaria):

1. Ampliar la formación académica

2. Potenciar el desarrollo personal y social

3. Nuevos contenidos relevantes

4. Conocimientos regionales

5. Proyectos de impacto social

La asignatura de Geografía está dentro del campo Exploración y comprensión del mundo natural y social. En preescolar se denomina Exploración y conocimiento del mundo; en primaria Exploración de la naturaleza y la sociedad (primer y segundo grado), La entidad donde vivo (tercer grado), Geografía (cuarto, quinto y sexto grado), y en secundaria Geografía de México y el mundo (primer grado), en segundo y tercero de secundaria se deja de impartir.

Los estudiantes deben comprender cómo está conformado el espacio geográfico y sus componentes, por ello, deben valorar su importancia, diversidad, y establecer relaciones que permitan ampliar y aplicar sus aprendizajes, requiriendo desarrollar conceptos, habilidades y actitudes espaciales. Los conceptos geográficos representan a los objetos que conforman el espacio, las habilidades implican acciones que favorecen la formación del conocimiento y las actitudes es el comportamiento que los estudiantes manifiestan a partir de la integración de los conocimientos y las habilidades.

Además, es importante mencionar que el tiempo establecido para la impartición de esta asignatura varía dependiendo del grado: 3 horas para primer y segundo en Exploración de la Naturaleza y la Sociedad; 4 horas para tercero en La entidad donde vivo, y 1.5 horas para cuarto, quinto y sexto grado en Geografía; puede verse una reducción significativa de esta materia en comparación con otras como español (8 horas) y matemáticas (6 horas), a las cuales siempre se les ha considerado de mayor relevancia. En secundaria se incrementa a 5 horas semanales en primer grado y desaparece en segundo y tercer grado. Esto indica que no se le da igual importancia a esta materia frente a otras. Para la impartición de esta asignatura se recomienda la utilización de los siguientes recursos didácticos (SEP, 2011b):
a) Imágenes geográficas.
b) Material cartográfico. 
c) Tecnologías de información y comunicación.

d) Recursos audiovisuales.

e) Libros y publicaciones periódicas.

f) Estadísticas y gráficas.

g) Reproducciones a escala.

h) Visitas escolares.

A pesar de las recomendaciones no es habitual que el docente utilice todos los recursos didácticos, y en el caso de las TIC no siempre brindan los resultados esperados, debido a que los profesores tienen que adaptar los contenidos a la tecnología digital disponible, con la posibilidad de no atender las necesidades del alumno porque los docentes no tienen la capacitación adecuada y no son útiles para la impartición de la asignatura de Geografía.

\section{Metodología}

En esta investigación, realizada durante todo el ciclo escolar 2018-2019, se utilizaron las técnicas de investigación necesarias de la metodología cualitativa y cuantitativa, es decir, de un enfoque mixto entendido como el conjunto de procesos sistemáticos, empíricos y críticos de investigación e implican la recolección y el análisis de datos cuantitativos y cualitativos, así como su integración y discusión conjunta (Hernández, Fernández y Baptista, 2014).

Dichas técnicas se emplearon para analizar el estudio de la asignatura de Geografía en nivel primaria y conocer las prácticas pedagógicas que se llevan a cabo durante el proceso de enseñanza y de aprendizaje de la asignatura Geografía impartida a los alumnos de cuarto, quinto y sexto grado de primaria en México, así como las estrategias y recursos utilizados.

Los métodos cualitativos utilizados fueron:

a) Análisis documental de los planes y programas de estudio, libro de Geografía, publicaciones previas, etc.

b) Observación en el contexto de estudio de los alumnos (aula de clase).

c) Entrevistas abiertas a los maestros sobre los contenidos y estrategias didácticas empleadas en el aula.

Y los métodos cuantitativos:

a) Encuesta a los alumnos.

En cuanto a la investigación documental tanto impresa como digital, se revisaron los planes y programas de estudio, el modelo educativo en el que está basado el sistema mexicano, el libro de Geografía para conocer los aspectos relacionados y una de las evaluaciones que se han realizado a nivel nacional a esta asignatura.

Posteriormente se definió la población de estudio, la cual consistió de 126 estudiantes de los grupos $\mathrm{A}$ y $\mathrm{B}$ de $4^{\circ}, 5^{\circ}$ y $6^{\circ}$ grado de nivel primaria perteneciente a una 
institución privada ubicada en la ciudad de Mazatlán, Sinaloa, México, y de las seis profesoras de los mencionados grupos; las edades de los alumnos oscilan entre los 8 y 12 años de edad. En la tabla 1 se muestra la distribución de los discentes sujetos de estudio por género y grado.

Tabla 1. Distribución de los sujetos de estudios de acuerdo a género y grado.

\begin{tabular}{|c|c|c|c|c|c|}
\hline \multirow[b]{3}{*}{ Grado } & \multicolumn{4}{|c|}{ Grupos } & \multirow[b]{3}{*}{ Total } \\
\hline & \multicolumn{2}{|c|}{ A } & \multicolumn{2}{|c|}{ B } & \\
\hline & Niñas & Niños & Niñas & Niños & \\
\hline Cuarto & 12 & 10 & 13 & 12 & 47 \\
\hline Quinto & 5 & 11 & 7 & 9 & 32 \\
\hline Sexto & 12 & 13 & 12 & 10 & 47 \\
\hline Total & \multicolumn{2}{|c|}{63} & \multicolumn{2}{|c|}{63} & 126 \\
\hline
\end{tabular}

Fuente: Construcción personal.

Una vez definido el objeto de estudio se empleó la técnica de la observación de cada uno de los grupos para conocer la forma en que se desenvuelven los sujetos mientras es llevada a cabo la práctica docente en la impartición de esta asignatura. Rojas (2011) menciona que esta técnica ofrece la ventaja de presenciar las sesiones de clase, seleccionar aquellas en que realmente se dan las interacciones, al mismo tiempo que permite tomar las secuencias de sentido (partes en que inicia, se desarrolla y culmina un tema) dentro de los registros.

Cabe mencionar que no se tomaron en cuenta los grados de primero, segundo y tercero porque en los dos primeros el contenido de la asignatura considera el entorno donde se desenvuelven (entorno familiar y local), y en tercero, aspectos relacionados con la entidad federativa a la que pertenece cada escuela; es a partir del cuarto grado que los contenidos impartidos son a nivel nacional.

Después de haber realizado la observación en el espacio del aula y recopilado los datos, se procedió a diseñar y aplicar una encuesta a cada uno de los alumnos para contrastar y tener más información y analizarla cuantitativamente; este instrumento constó de diez preguntas.

Finalmente se entrevistó tanto a docentes como a alumnos para conocer de viva voz la experiencia de los sujetos involucrados en el proceso de enseñanza y de aprendizaje de esta asignatura en el espacio del aula.

\section{Resultados}

A través del análisis de los documentos, el cual consistió en el procesamiento y recuperación de información contenida en documentos, independientemente del soporte en que se encontraran (Modelo Educativo para la Educación Obligatoria, 
Plan de Estudios 2011 de Educación Básica y el libro de texto de la materia), en tales archivos se conoció el objetivo de los planes y programas, el mapa curricular del nivel básico, los estándares curriculares, perfiles de egresos y competencias esperadas y los contenidos de la asignatura de Geografía. Se realizó una revisión de las evaluaciones históricas efectuadas por la SEP a la mencionada materia y se encontró que, desde el año 2006, los niños mexicanos han sido medidos con la Evaluación Nacional de Logro Académico en Centros Escolares (ENLACE), teniendo como propósito comparar los conocimientos y habilidades que tienen los alumnos.

Tabla 2. Resultados de prueba Enlace Básica - Geografía 2011 para alumnos de $3^{\circ}$ a $6^{\circ}$ de primaria.

\begin{tabular}{cc}
\hline Nivel de logro & Geografía \\
\hline Insuficiente & $12.4 \%$ \\
\hline Elemental & $53.7 \%$ \\
\hline Bueno & $29.9 \%$ \\
\hline Excelente & $4.0 \%$ \\
\hline
\end{tabular}

Fuente: Construcción personal con datos de la SEP (2011d).

Como se muestra en la tabla 2, en el año 2011 se aplicó la prueba ENLACE considerando tres materias: Español, Matemáticas y Geografía, y por su aprovechamiento, los estudiantes de tercero, cuarto, quinto y sexto grado; en la última asignatura se puede observar que en su mayoría se encuentran en el nivel de logro "elemental", es decir, los discentes realizan inferencias sencillas sobre los estados, capitales y biodiversidad; establecen relaciones entre dos o más elementos, generalmente les es muy difícil dar una imagen clara de una idea si se usan solamente palabras habladas o escritas.

Cabe mencionar que esta prueba ya no es aplicada, pero se tomó como referencia para comparar los resultados anteriores con los actuales. De acuerdo a la SEP (2017), el bajo desempeño de la mayoría de los estudiantes mexicanos se ha evidenciado de manera reiterada en distintas pruebas, tanto nacionales (ExCALE/ENLACE/PlANEA) como internacionales (PISA).

Al utilizar la técnica de observación no participativa de los sujetos de estudio se pudo conocer cómo se desenvuelven oral y corporalmente, la relación entre sus compañeros y profesor, los productos elaborados durante la clase, así como las habilidades y dificultades mostradas en el proceso de aprendizaje de los alumnos; esto permitió conocer, describir, comprender y registrar el fenómeno observado. La interacción fue realizada de manera natural mas no intrusiva, es decir, tratando de no ejercer un 
control físico o romper con la estructura normal del escenario en el que se desenvolvían. Los datos obtenidos fueron registrados y analizados para con ellos estructurar y formular preguntas para integrarlas a las encuestas y las entrevistas y obtener así más datos. Ambas técnicas se aplicaron en otro momento para no interferir con el escenario observado, que se estaba desarrollando en tiempo real.

A través de la técnica de la observación se conoció a los sujetos (alumnos y maestros) dentro de su contexto, conociendo la realidad tal y como ellos la perciben. Por otro lado, se analizó el entorno, es decir, el espacio donde ellos pasan largas jornadas de tiempo y donde se realizan las prácticas de enseñanza y de aprendizaje, con el objetivo de que los conocimientos adquiridos en esos espacios sean utilizados en algún futuro. Se pudo observar que el espacio donde ellos interaccionan se encontraba decorado con textos, mapas y figuras alusivas a ciertos temas.

Se pudo constatar que en las clases de Geografía tanto alumnos como docentes utilizan el libro de texto como guía principal durante el proceso de enseñanza y de aprendizaje avanzando al ritmo del docente, la participación de los alumnos es controlada conforme avanza el docente en el tema, teniendo así un ritmo homogéneo.

Los pupitres donde pasan largas jornadas se encuentran distribuidos en 4 o 5 filas, dependiendo del grado y del número de los alumnos, asignado uno a cada alumno. Estos tienen un espacio para guardar sus materiales y libros que no utilizan o aquellos que necesitan frecuentemente. Hay un mueble de madera dividido en secciones, donde cada niño tiene un espacio (locker) para colocar sus libros y cuadernos; el salón posee una pizarra de color blanco, la cual es empleada con plumones de colores de agua, hay un escritorio y una silla asignada para el maestro, así como un locker especialmente para él, para guardar sus cosas y materiales para el uso de la clase, como plumones, diccionarios, libros, cuadernos, lápices, plumas, listas de asistencia, etc.

Como complemento a esta técnica se realizó la encuesta; algunas de las preguntas realizadas estaban relacionadas con las horas dedicadas a la semana para el estudio de los contenidos de la materia, opinión de los alumnos sobre la asignatura, utilidad que le encuentran, opinión sobre las estrategias, frecuencia con que utilizan el libro y el cuaderno, dificultad para aprender los contenidos, uso de la computadora, frecuencia del fomento de esta materia en casa.

Los resultados a la interrogante "Horas dedicadas a la semana a la asignatura Geografía, en el aula" se resumen en la figura 1. Las respuestas se concentran en las opciones 3 a 4 horas y 5 horas o más; por lo que nadie contestó a las opciones 0 horas y 1 a 2 horas. 47 de los 126 encuestados, el 37\%, afirman dedicarle entre 3 y 4 horas a la asignatura Geografía, mientras que 79 sujetos, el 63\%, dicen dedicar 5 o más horas a la materia. 


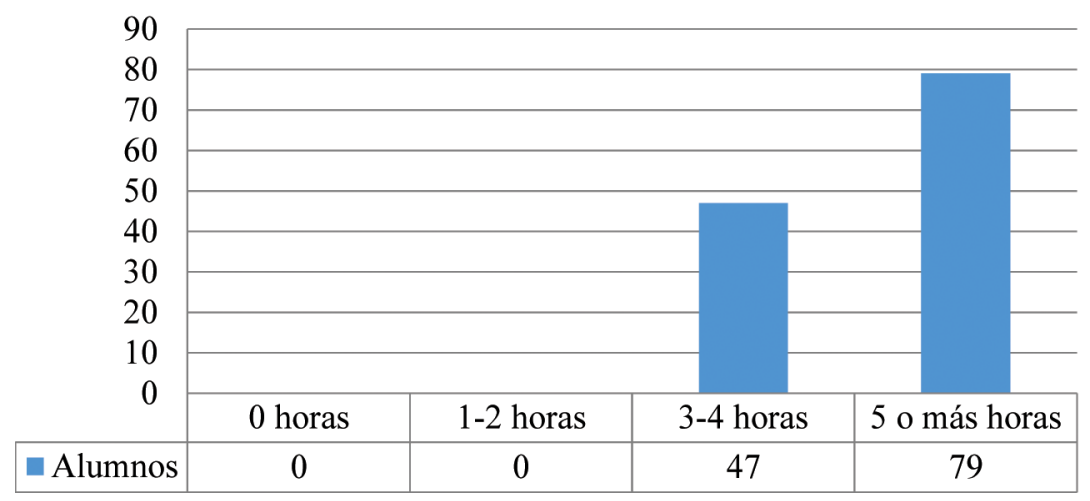

Figura 1. Horas dedicadas a la semana a la asignatura Geografía, en el aula. Fuente: Construcción personal.

Los resultados a la pregunta "Opinión de los alumnos sobre la asignatura de Geografía" se resumen en la figura 2. Las respuestas se concentran en la opción Interesante. 25 de los 126 encuestados, el 19.8\%, afirman que la Geografía es Muy interesante; 16 sujetos, el $12.7 \%$, la consideran Poco interesante; a 14 sujetos, el 11.1\%, les resulta Nada interesante; mientras que 71 sujetos, el 56.3\%, dicen que la materia es Interesante.

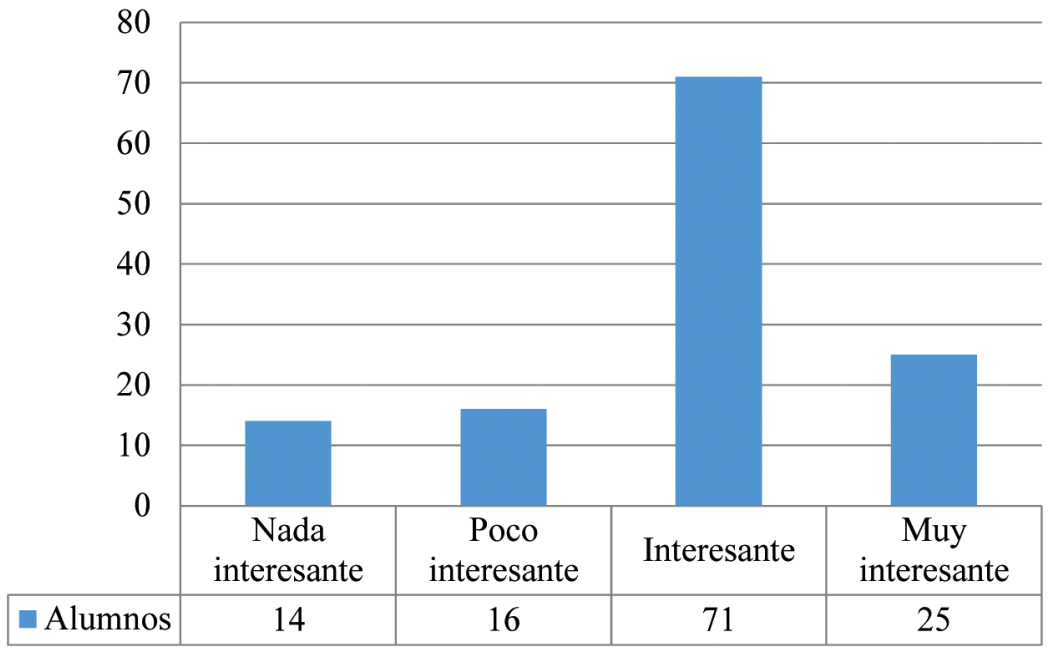

Figura 2. Opinión de los alumnos sobre la asignatura Geografía.

Fuente: Construcción personal.

Cuando se les cuestionó sobre la utilidad percibida de la asignatura de Geografía por los alumnos, los encuestados respondieron como se indica en la figura 3. Las respuestas se concentran en la opción Bastante, 91 sujetos seleccionaron esta opción, el 72\%. Que tiene Mucha utilidad fue elegida por 18 de los 126 encuestados, el 14\%; mientras que 10 niños, el 7.9\%, consideran que la Geografía es de Poca utilidad, y 7 alumnos, el 5.6\%, dicen que optaron por la opción Nada. 


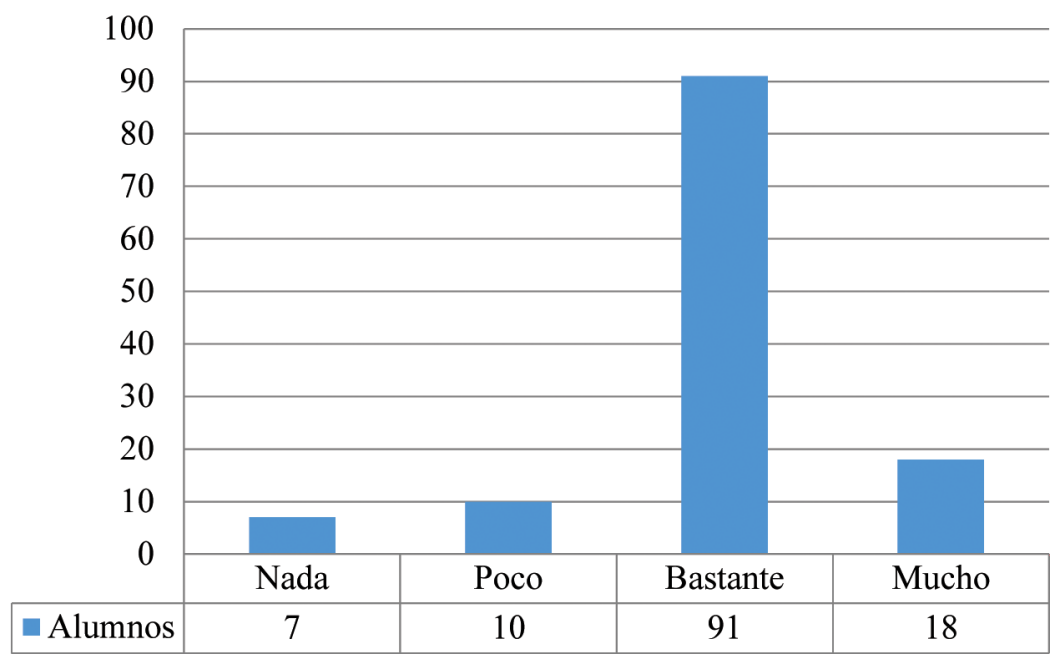

Figura 3. Opinión de los alumnos sobre la utilidad de la asignatura de Geografía en su vida. Fuente: Construcción personal.

Los resultados a la interrogante "Opinión sobre las estrategias utilizadas por el maestro, en el aula" se resumen en la figura 4. La mayoría de las respuestas se concentran en las opciones Poco Interesante e Interesante, siendo la primera la elegida por 69 de los 126 encuestados, el 54.8\%; 48 sujetos, el 38\%, afirman que la estrategia es Interesantes. 5 alumnos, el 4\%, consideran que es Muy interesante, y 4 niños, el 3\%, optaron por Nada interesante.

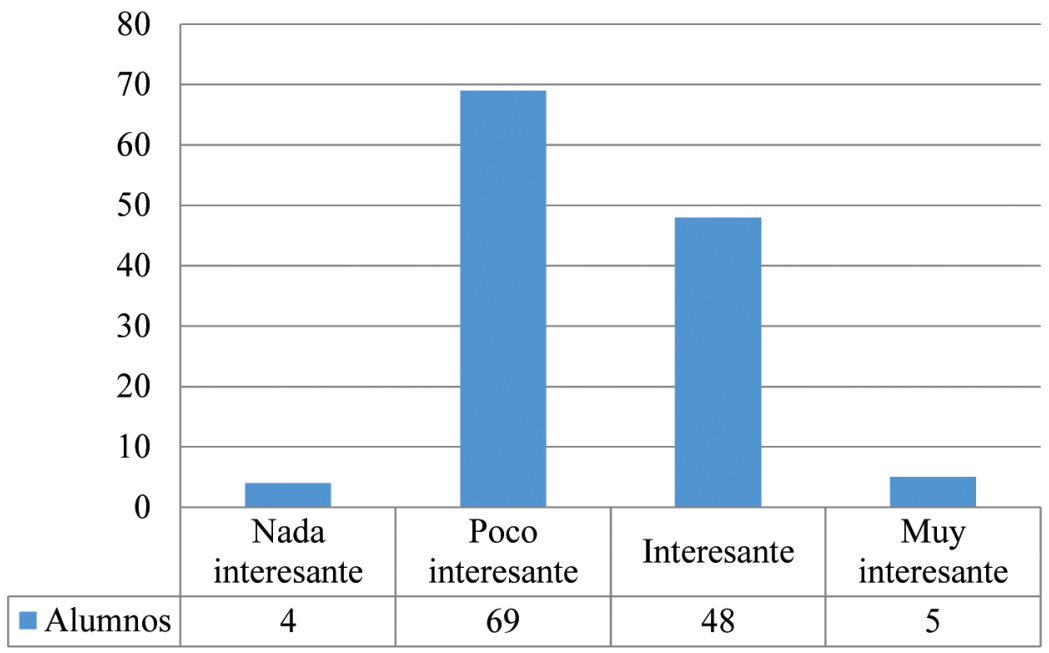

Figura 4. Opinión de los alumnos a las estrategias utilizadas por el maestro en clase. Fuente: Construcción personal.

Al cuestionárseles sobre el uso del libro de texto y el cuaderno, en el aula, los sujetos de estudio contestaron según se observa en la figura 5. Las respuestas se concentran en las opciones Siempre y Casi siempre; nadie contestó a las opciones Pocas 
veces y Nunca. 15 de los 126 encuestados, el 11.9\%, afirma que Casi siempre utilizan tanto el libro de texto como el cuaderno, mientras que 111 sujetos dicen que Siempre.

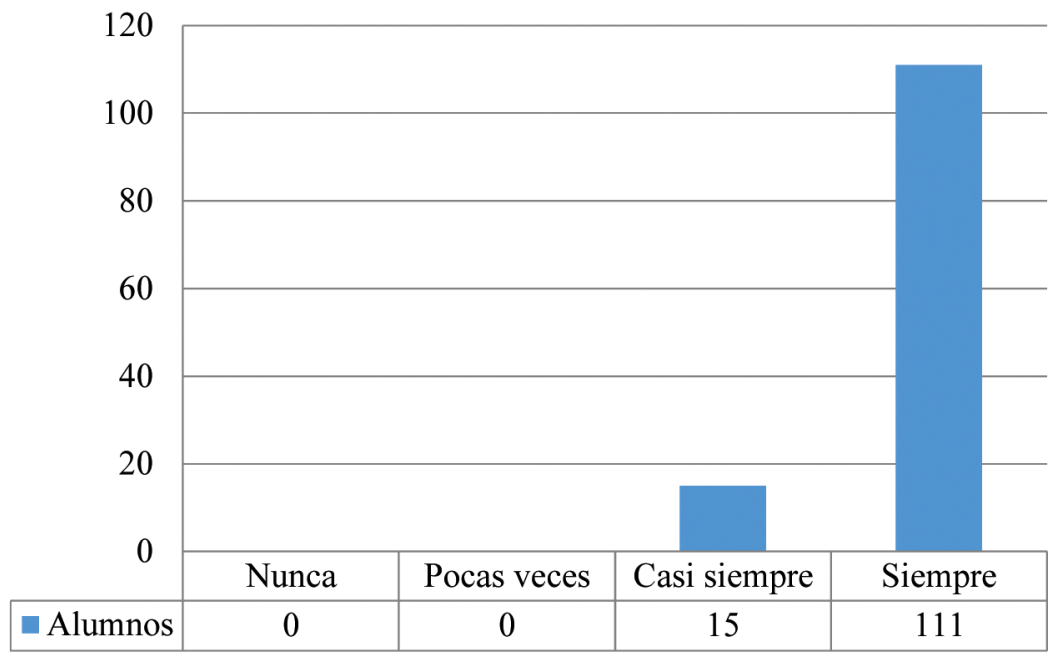

Figura 5. Frecuencia con que se utiliza el libro de texto y el cuaderno en clases.

Fuente: Construcción personal.

Los resultados a la interrogante "Dificultad para aprender los contenidos de la asignatura de Geografía, en el aula" se resumen en la figura 6. Las respuestas se concentran en la opción Mucho. 26 de los 126 encuestados, el 20.6\%, afirman que la dificultad de Geografía es Poca; 11 sujetos, el 8.7\%, consideran que Nada, y 11 sujetos, el $8.7 \%$, indican que Demasiado, mientras que 78 sujetos, el $61.9 \%$, dicen que se les dificulta Mucho.

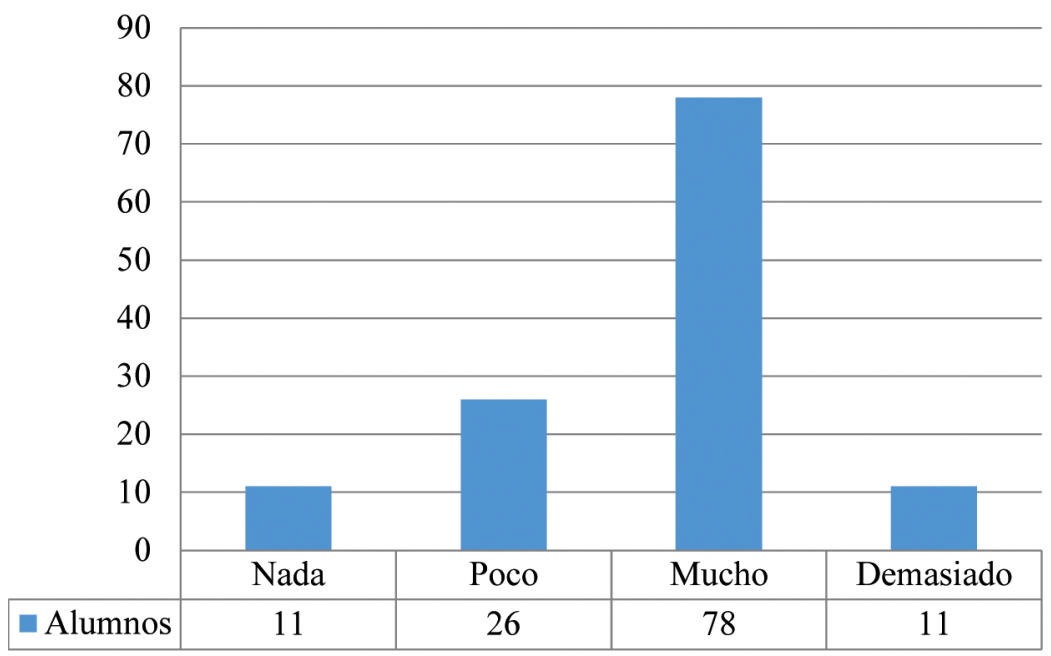

Figura 6. Dificultad para aprender los contenidos de la asignatura de Geografía.

Fuente: Construcción personal. 
Los resultados a la pregunta "Conocimientos de los alumnos en el manejo de las computadoras" se resumen en la figura 7. Las respuestas se concentran en Demasiado, Mucho y Poco; nadie contestó a la opción Nada. 25 de los 126 encuestados, el 19.8\%, afirman tener Mucho conocimiento y 3 sujetos, el 2.4\%, consideran que poseen Poco, mientras que 98 sujetos, el 77.8\%, dicen el conocimiento en el uso de las computadoras es Demasiado.

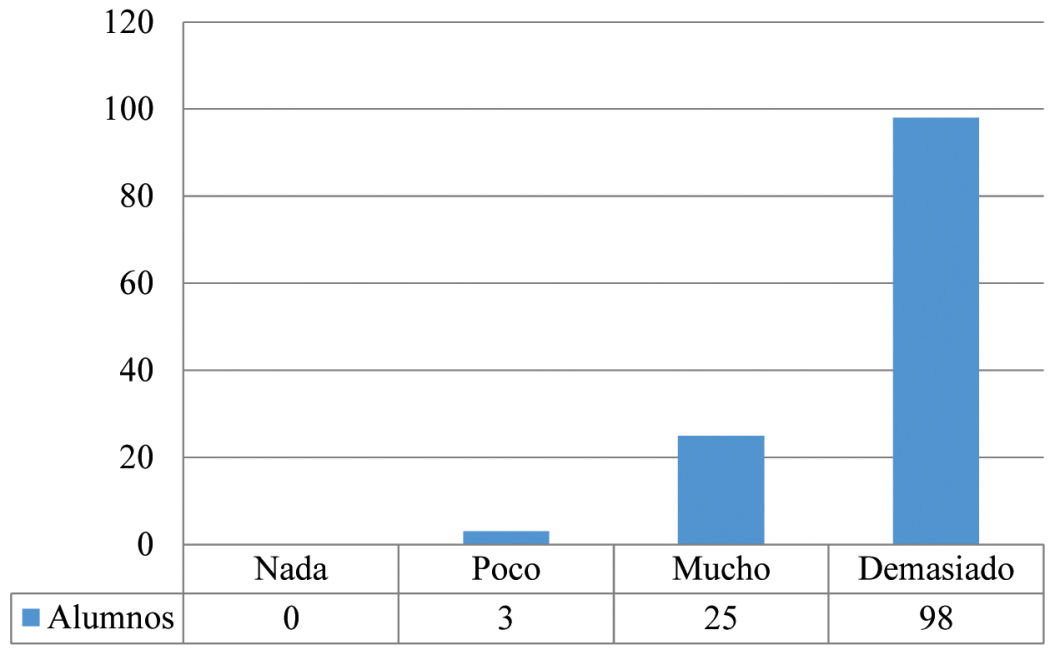

Figura 7. Conocimientos de los alumnos en el uso de las computadoras.

Fuente: Construcción personal.

Las respuestas a la interrogante "Días que utilizan la computadora para ver los contenidos de la asignatura de Geografía" se concentran en las opciones 1 o 2 veces; nadie contestó a las opciones Ninguna, 3 o 4 veces y 5 o más veces. Los 126 encuestados, el 100\%, afirman que hacen uso de la computadora 1 o 2 veces a la semana en la asignatura de Geografía.

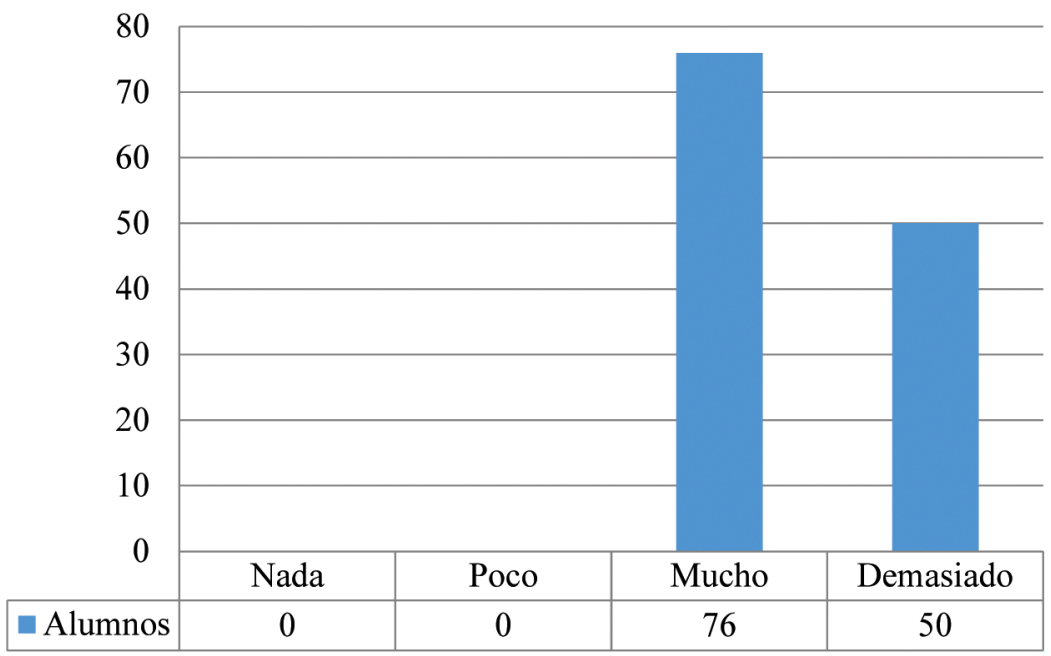

Figura 8. Motivación de los alumnos para estudiar Geografía a través de un software.

Fuente: Construcción personal. 
Los resultados a la interrogante "Motivación para estudiar los contenidos de Geografía a través de un software educativo" se resumen en la figura 8. Las respuestas se concentran en las opciones Demasiado y Mucho; nadie contestó las opciones Nada y Poco. 50 de 126 encuestados, el 39.7\%, afirman que les motivaría Demasiado, mientras que 76 sujetos, el 6.3\%, dicen tener Mucha motivación.

Los resultados a la interrogante acerca del fomento de los contenidos de la asignatura de Geografía, en casa, se resumen en la figura 9. Las respuestas se concentran en las opciones Nunca y Pocas veces; nadie contestó Muchas veces y Siempre. 106 de los 126 encuestados, el 84.1\%, afirman que en casa Nunca se les fomenta sobre la Geografía, mientras que 20 sujetos, el $84.1 \%$, dicen que Pocas veces se les habla sobre la asignatura.

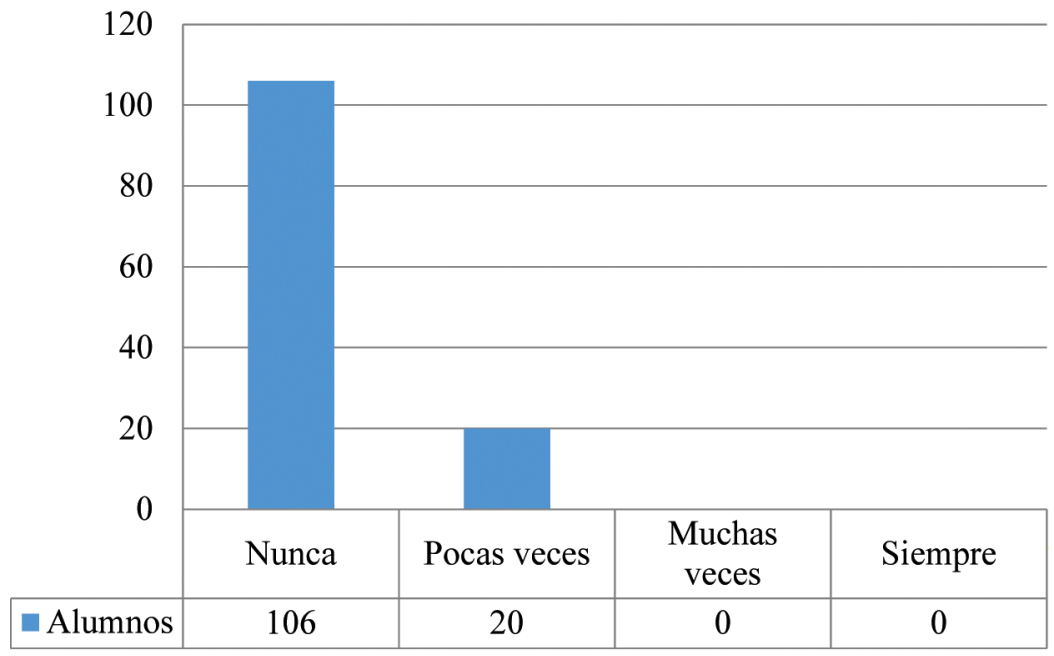

Figura 9. Frecuencia con la que se fomenta la asignatura de Geografía en casa. Fuente: Construcción personal.

Posterior a la encuesta, se entrevistó a los 126 estudiantes $\left(4^{\circ}, 5^{\circ}\right.$ y $6^{\circ}$ grados) y 6 docentes para recopilar datos que no fue posible recabar con las otras técnicas. Los alumnos expresaron que el tiempo asignado a la materia Geografía es escaso con respecto a otros cursos; sus contenidos les resultan interesantes, sin embargo, casi siempre utilizan el libro de texto, el cuaderno, mapas y láminas como apoyo a los contenidos. También afirman que les gustaría utilizar otros recursos como las computadoras, pues los discentes saben manejarlas, debido a que desde los primeros grados los han capacitado en la clase de computación y en casa tienen acceso a ellas. Lamentablemente en el hogar no se les fomentan los contenidos de la asignatura, solo cuando tienen tarea de esta materia.

Los docentes comentaron que el tiempo planificado para Geografía es el establecido en los planes y programas de la SEP, debido a que deben cubrir todos los contenidos de cada una de las materias y ninguna debe interferir con las demás. 
Es frecuente que la información contenida en los libros no se revise a fondo, pues consideran que el tiempo del ciclo escolar es justo para la realización de todas las actividades académicas, culturales y deportivas calendarizadas, y tienen que cumplir con el resto de los temas establecidos en la planeación. El centro de cómputo rara vez es utilizado por los docentes, entre otras cosas porque la demanda del mismo es alta, además tienen que adecuar los contenidos de la materia a los recursos disponibles en la computadora y no al revés.

\section{Conclusiones}

El propósito de esta investigación fue analizar el proceso de enseñanza y de aprendizaje de la asignatura Geografía en nivel primaria, tomándose como muestra una escuela privada de la ciudad de Mazatlán, Sinaloa, México, y como sujetos de estudio a los alumnos de $4^{\circ}, 5^{\circ}$ y $6^{\circ}$ grados.

Los resultados arrojados en esta investigación permiten comprender el contexto del proceso de enseñanza y de aprendizaje, base para la etapa de análisis del desarrollo de un software, y un futuro diseño y desarrollo, que cuente con las funciones y características requeridas por los alumnos y maestros, y además sirva como una estrategia tecnológica para la asignatura de Geografía para apoyar en el proceso de enseñanza, proceso de aprendizaje, desarrollo y fortalecimiento de las competencias.

El objetivo fue cumplido en su totalidad, con apoyo de las técnicas de análisis documental, observación, entrevista y encuesta. La muestra consistió de 126 alumnos de $4^{\circ}$ a $6^{\circ}$ grados, 63 niños y 63 niñas, y seis profesoras, quienes fueron observados durante todo el ciclo escolar 2018-2019.

Para la asignatura de Geografía los docentes utilizan los materiales didácticos recomendados y proporcionados por la SEP, además de otro material de apoyo, pero consideran que no se aprovechan a cabalidad debido a que el tiempo asignado a la materia no es suficiente para analizar los materiales, además que de considerarlos como atractivos y motivantes para los niños. Los profesores afirman que están dispuestos a adaptar los contenidos de Geografía a las tecnologías digitales, a pesar del esfuerzo extra que ello significa, pero se los impide la poca disponibilidad horaria del centro de cómputo, el cual frecuentemente está saturado por otros docentes y grupos de estudiantes.

Por su parte, los discentes expresan que dedican tiempo a la asignatura, tanto en el aula como en sus hogares en la realización de tareas; la mayoría la considera interesante y útil, pero a la mayoría les resulta poco interesante, quizá por el hecho de que se utilicen estrategias tradicionales como el libro de texto y el cuaderno. También afirmaron que se les dificulta mucho el aprendizaje de la geografía; 123 de los 126 alumnos dicen tener muchos o demasiados conocimientos de manejo de computadora, y los 126, el 100\%, afirman utilizar la computadora 1 o 2 veces por semana para 
estudiar geografía. El 100\% indica que tendrían mucha o demasiada motivación por estudiar geografía por medio de un software, aunque los 126 estudiantes dicen que nunca o pocas veces se les fomenta el estudio de la geografía en el hogar.

El uso del centro de cómputo para el estudio de la geografía, el estímulo a los docentes para que adapten los contenidos de la asignatura a las nuevas tecnologías, así como el uso de un software diseñado específicamente para este propósito, además del fomento del estudio de la Geografía en casa por parte de los padres, serían adecuados alicientes para expandir el conocimiento en esta materia. De acuerdo con Walczak y Taylor (2018), no existe una diferencia estadísticamente significativa entre los dos métodos de enseñanza (basados en TIC y tradicionales), pero la combinación de los dos puede conducir a mejoras significativas en los resultados del aprendizaje. También se necesita investigación para ciertas clases de aplicaciones educativas, incluidas geometría y geografía (Larkin, 2016).

\section{REFERENCIAS}

Blankman, M., Schoonenboom, J., Van der Schee, J., Boogaard, M., y Volman, M. (2016). Learning to teach geography for primary education: results of an experimental programme. Journal of Geography in Higher Education, 40(3), 425-441. doi: 10.1080/03098265.2016.1144731.

Castañeda, J. (2005). La enseñanza de la geografía en México. Una visión histórica; 1821-2000. Ponencia presentada en Análisis de Encuentro de Geógrafos de América Latina. Sao Paulo, Brasil. Recuperado de: http:// www.observatoriogeograficoamericalatina.org.mx/ egal10/Ensenanzadelageografia/Investigacionydesarrolloeducativo/08.pdf.

Hernández, R., Fernández, C., y Baptista, P. (2014). Metodología de la investigación (6a. ed.). Ciudad de México: McGraw-Hill.

Larkin, K. (2016). Geometry and iPads in primary schools: Does their usefulness extend beyond tracing an oblong? En P. S. Moyer-Packenham (ed.), International perspectives on teaching and learning mathematics with virtual manipulatives (pp. 247-274). Springer International Publishing. doi: 10.1007/9783-319-32718-1_11.

Lima, L., Bonilla, F., y Arista, V. (2010). La enseñanza de la historia en la escuela mexicana. Proyecto Clío 36, (s.n.), 1-16. Recuperado de: http://clio.rediris. es/n36/articulos/limaetalii.pdf.
Martin, F. (2006). Teaching geography in primary schools learning to live in the World. Cambridge, Inglaterra: Chris Kington Publishing.

Prensky, M. (2001). Digital natives, digital immigrants. On the Horizon, 9(5), 1-6. doi: https://doi. org/10.1108/10748120110424816.

Rojas, I. R. (2011). Elementos para el diseño de técnicas de investigación: una propuesta de definiciones y procedimientos en la investigación científica. Tiempo de Educar, 12(24), 277-297. Recuperado de: https://www.redalyc.org/articulo. oa?id=311/31121089006.

Santillán, M. (2006). Tecnologías de la información y de la comunicación en la educación. Revista Mexicana de Investigación Educativa, 11(28), 7-10. Recuperado de: http:// comie.org.mx/revista/v2018/rmie/index. $\mathrm{php} / \mathrm{nrmie} /$ article/view/669/669.

SEP [Secretaría de Educación Pública] (2011a). Plan de estudios 2011. Educación Básica. Ciudad de México: SEP. Recuperado de: https://www.gob.mx/ cms/uploads/attachment/file/20177/Plan_de_ Estudios_2011_f.pdf.

SEP (2011b). Programas de estudio 2011. Guía para el maestro. Educación Básica. Primaria. Cuarto Grado. Ciudad de México: SEP. Recuperado de: https:// sector2federal.files.wordpress.com/2012/05/4programa-cuarto-grado-2011.pdf. 
SEP (2011c). Los retos de la geografía en educación básica. Ciudad de México: SEP. Recuperado de: http:/ / edu. jalisco.gob.mx/cepse/sites/edu.jalisco.gob.mx.cepse/ files/los_reto_de_la_geografia._en_educacion_basica._su_ensenanza_y_aprendizaje.pdf.

SEP (2011d). Resultados prueba ENLACE 2011. Básica y Media Superior. Ciudad de México: SEP. Recuperado de: https://staticmx.unoi.com/unoinews/2011/09/ ENLACE2011_versionFinalSEP.pdf.

SEP (2017). Modelo educativo para la educación obligatoria. Ciudad de México: SEP. Recuperado de: https:/ / www. gob.mx/cms/uploads/attachment/ file/207252/ Modelo_Educativo_OK.pdf.
Tomcikova, I., y Rakytova, I. (2018). Geography of local landscape in the educational process in primary schools in Slovakia. European Journal of Geography, 9(1), 49-61. Recuperador de: http:/ / www.eurogeographyjournal.eu/articles/GEOGRAPHY\%20OF\%20 LOCAL\%20LANDSCAPE\%20IN\%20THE\%20 EDUCATIONAL\%20PROCESS\%20IN\%20PRI-

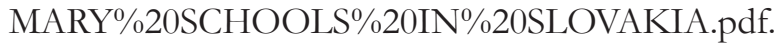
Walczak, S., y Taylor, N. G. (2018). Geography learning in primary school: Comparing face-to-face versus tabletbased instruction methods. Computers \& Education, 1172018), 188-198. doi: https://doi.org/10.1016/j. compedu.2017.11.001.

Cómo citar este artículo:

Bernal Díaz, M. A., Fonseca Polanco, D. C., y Zaldívar Colado, Á. (2021). Análisis del proceso de enseñanza y de aprendizaje de los contenidos de la asignatura de Geografía en nivel primaria en México. IE Revista de Investigación Educativa de la REDIECH, 12, e943. doi: https://doi.org/10.33010/ie_rie_rediech.v12i0.943. 